\title{
Transport and Thermoelectric Properties of Magnetic Organic Structures
}

\author{
K. ZBERECKI* AND R. SWIRKOWICZ \\ Faculty of Physics, Warsaw University of Technology, ul. Koszykowa 75, 00-662 Warsaw, Poland \\ Using ab initio methods, we study transport and thermoelectric phenomena of magnetic organic chains func- \\ tionalized with nitroxide groups. We predict very good thermoelectric performance of the structure, as the con- \\ ventional and spin Seebeck coefficients are remarkably enhanced. Our results suggest that magnetic organic chains \\ would have a great potential for applications in spintronic devices.
}

DOI: 10.12693/APhysPolA.133.532

PACS/topics: 73.63.-b, 75.75.-c

\section{Introduction}

Spin Seebeck effect, which is a spin analog of the well-known Seebeck effect, can be observed in magnetic nanostructures and corresponds to heat conversion into spin voltage $\Delta V_{s}$. Quantitatively the effect is described by the spin Seebeck coefficient $S_{s}=-\Delta V_{s} / \Delta T$, giving the spin voltage generated due to temperature gradient $\Delta T$. The phenomenon results from the interplay between charge, heat and spin transport and was observed in thin films, magnetic tunnel junctions and nanostructures [1-4]. Considerable values of $S_{s}$ were predicted in specially designed one-dimensional graphene-like systems, or functionalized carbon structures [5] as well as in quantum dots [6].

In the present paper we study spin effects in electron transport and spin thermoelectric phenomena of magnetic one-dimensional organic structures functionalized with radical nitroxide groups and demonstrate that the systems are half-metallic ferromagnets and can act as perfect spin filters as well as efficient generators of spin voltage. A variety of molecules and polymers with radical groups are known in organic chemistry. It was shown that the systems are stable and exhibit non-zero magnetic moment. High spin states of molecules and polymers result from ferromagnetic exchange coupling between unpaired electrons [7-10]. Thereby, such structures can be important in view of potential application in molecular electronics and spintronic devices [11].

\section{Electronic, transport and thermoelectric properties}

We consider several types of organic one-dimensional structures functionalized with radical nitroxide groups which are placed in different positions as presented in Fig. 1. The appropriate structures are denoted as I, II, III and IV, respectively. First, we investigate the

\footnotetext{
*corresponding author; e-mail: zberecki@if.pw.edu.pl
}

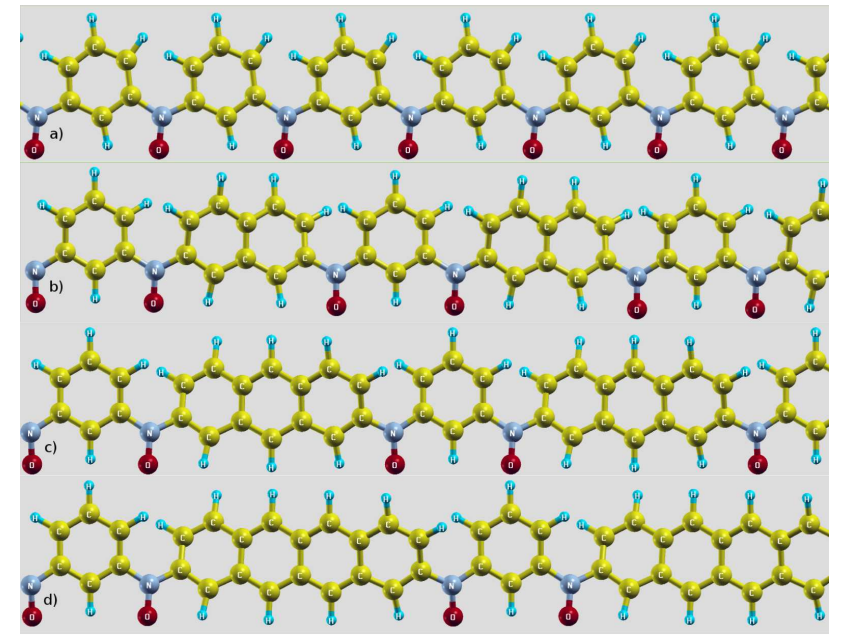

Fig. 1. Geometry of the structures - I (a), II (b), III (c) and IV (d).

band properties of the systems. Calculations were performed within density functional theory (DFT) with use of Siesta code $[12,13]$ and the atomic double-polarized basis (DZP) was applied. General gradient approximation (GGA) and PBE parameterization was used for exchange-correlation functional [14]. The structures under consideration were optimized until atomic forces converged to $0.02 \mathrm{eV} / \mathrm{A}$. Numerical calculations show that all the structures I-IV exhibit ferromagnetic ordering. Moreover, the systems are semiconductors with the energy gap close to $1 \mathrm{eV}$. The bands are strongly spinpolarized, especially the ones in the vicinity of the Fermi level, which are related to NO groups. The band structure of the system I is relatively simple with narrow spinpolarized bands just below and above the Fermi level (see our previous work [15]). These narrow bands are separated by the two wide gaps from the groups of low and high-lying states. In the case of structures II, III and IV appear additional, fully spin-polarized narrow bands lying in the gaps between $-1 \mathrm{eV}$ and $-2 \mathrm{eV}$, and also between $1 \mathrm{eV}$ and $2 \mathrm{eV}$. We present two examples of the band structure features obtained for systems II and III (Fig. 2a 

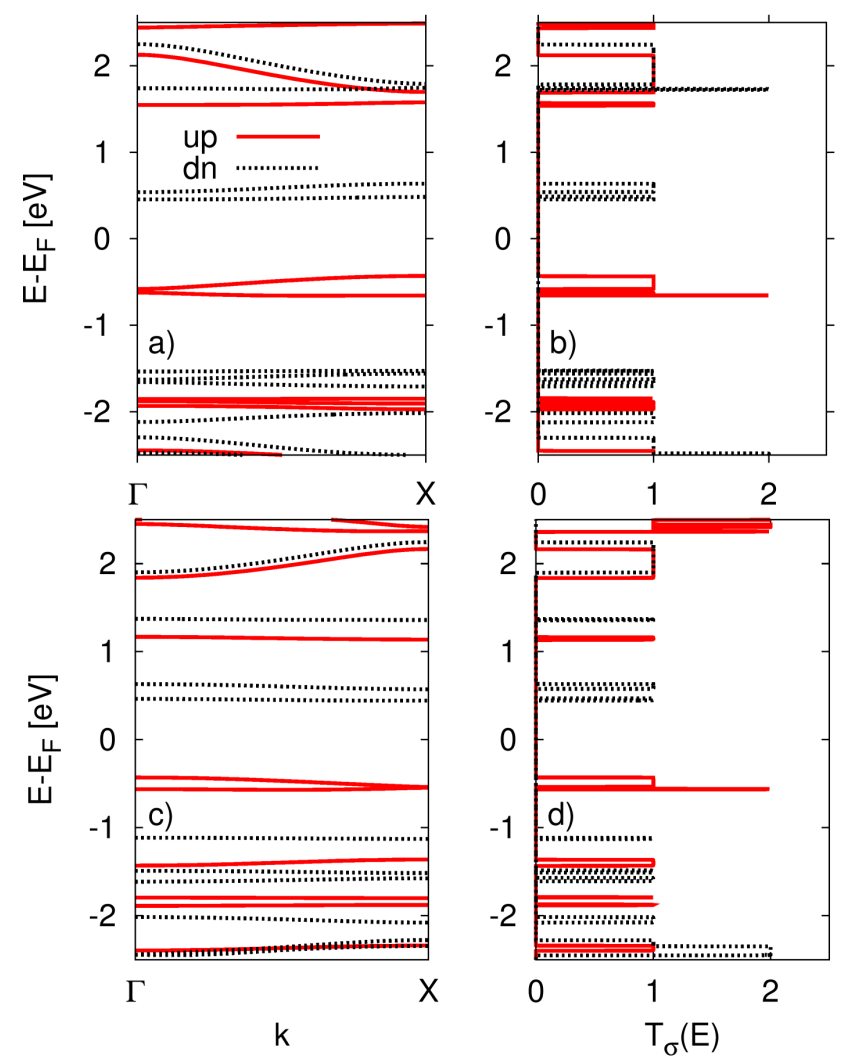

Fig. 2. Band structure and spin-resolved transmission function $T_{\sigma}(E)$ of the radical chains of types II and III. The energy is measured here from the Fermi energy $E_{\mathrm{F}}$ of the corresponding electrically neutral system.

and $2 \mathrm{c}$, respectively). The two wide gaps near $\pm 1 \mathrm{eV}$ can be well seen for structure II (Fig. 2a), whereas the appearance of additional narrow spin-polarized bands in the gaps is well demonstrated in Fig. 2c for structure III

The spin-resolved energy-dependent transmission function $T_{\sigma}(E)$ is also presented for structures II and III, Fig. $2 \mathrm{~b}$ and $2 \mathrm{~d}$, respectively. The transmission was determined within non-equilibrium Green function method (NEGF) as implemented in Transiesta code [14]. According to Fig. $2 \mathrm{~b}$ and $2 \mathrm{~d}$ it can be seen that flat spinpolarized bands lead to very narrow peaks in transmission function separated by the gaps. The number of these peaks increases in systems from I to IV.

To study transport properties we assume that a given carbon chain is attached to semi-infinite electrodes made from the same material. Transport is investigated in the linear response regime. Then, the spin-dependent electrical conductance is equal to: $G_{\sigma}=e^{2} L_{0 \sigma}$, whereas the spin Seebeck coefficient $S_{s}$, determined under the condition of vanishing charge and spin currents, is given by the following formula:

$$
S_{s}=-\frac{1}{2|e| T}\left(L_{1 \uparrow} / L_{0 \uparrow}-L_{1 \downarrow} / L_{0 \downarrow}\right) .
$$

In the above expressions $L_{n \sigma}=-\frac{1}{h} \int \mathrm{d} E T_{\sigma}(E)(E-$ $\mu)^{n} \frac{\partial f}{\partial E}$ with $n=0,1$ and $f(E-\mu)$ is the Fermi-Dirac distribution function corresponding to the chemical potential $\mu$ measured with respect to Fermi energy, and temperature $T$ equal in both electrodes.

First, we discuss the electrical conductance $G=G_{\uparrow}+$ $G_{\downarrow}$ in dependence on the chemical potential $\mu$, which could be tuned with use of gate voltage. The results obtained for structures I-IV are presented in Fig. 3 for temperatures $T=300 \mathrm{~K}$ and $T=200 \mathrm{~K}$ (inset).

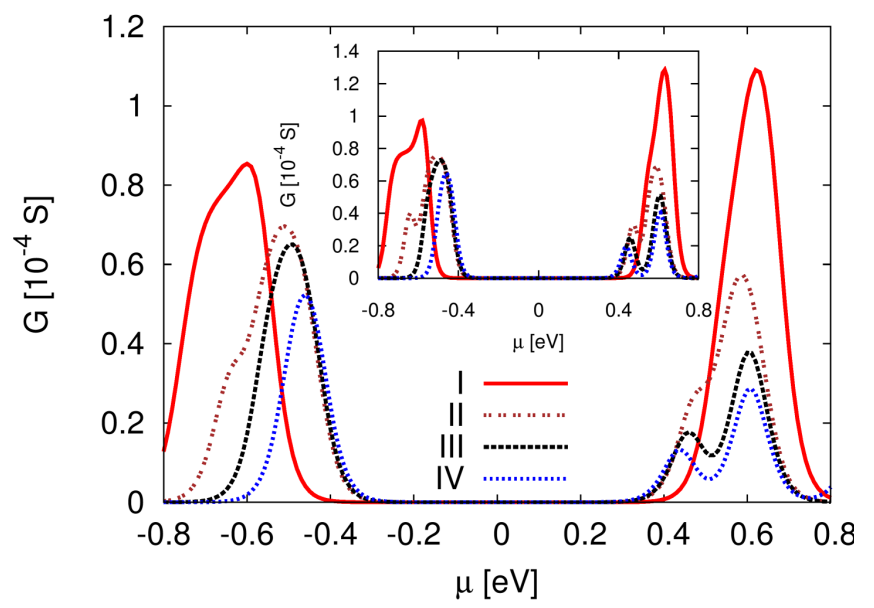

Fig. 3. Total electrical conductance for $T=300 \mathrm{~K}$ (in the inset shown for $T=200 \mathrm{~K}$ ) of radical chains.

For each type of the structure two well-defined peaks can be observed in the conductance, one lying in the region of negative and one for positive values of chemical potential. The height and the width of the peaks strongly depend on temperature, the conductance is suppressed when the temperature is increasing, what is the wellknown result. However, the conductance also strongly depends on the structure type. When the structure is changed from the type I to IV, the conductance is considerable lowered and moreover, the peak shift as well as the narrowing can be observed, especially for negative values of $\mu$. Such changes in the conductance are directly related to changes in the band structure near the Fermi level of the discussed chains. It is also important that peaks appearing in the conductance are fully spin polarized and correspond to spin up for $\mu<0$ and to spin down for positive values of $\mu$. Accordingly, the spin polarization $P=\frac{G_{\uparrow}-G_{\downarrow}}{G_{\uparrow}+G_{\downarrow}}$ is equal to +1 or -1 for negative and positive values of chemical potential, respectively. Accordingly, each of the systems can act as a perfect spin filter.

The spin Seebeck coefficient $S_{s}$ calculated in a function of chemical potential is presented in Fig. 4.

Different curves correspond to four different types of the structures from Fig. 1. The results strongly depend of the type of the chain. At elevated temperatures $(T=300 \mathrm{~K})$ contributions coming from both edges of energy gap overlap due to the considerable broadening of 

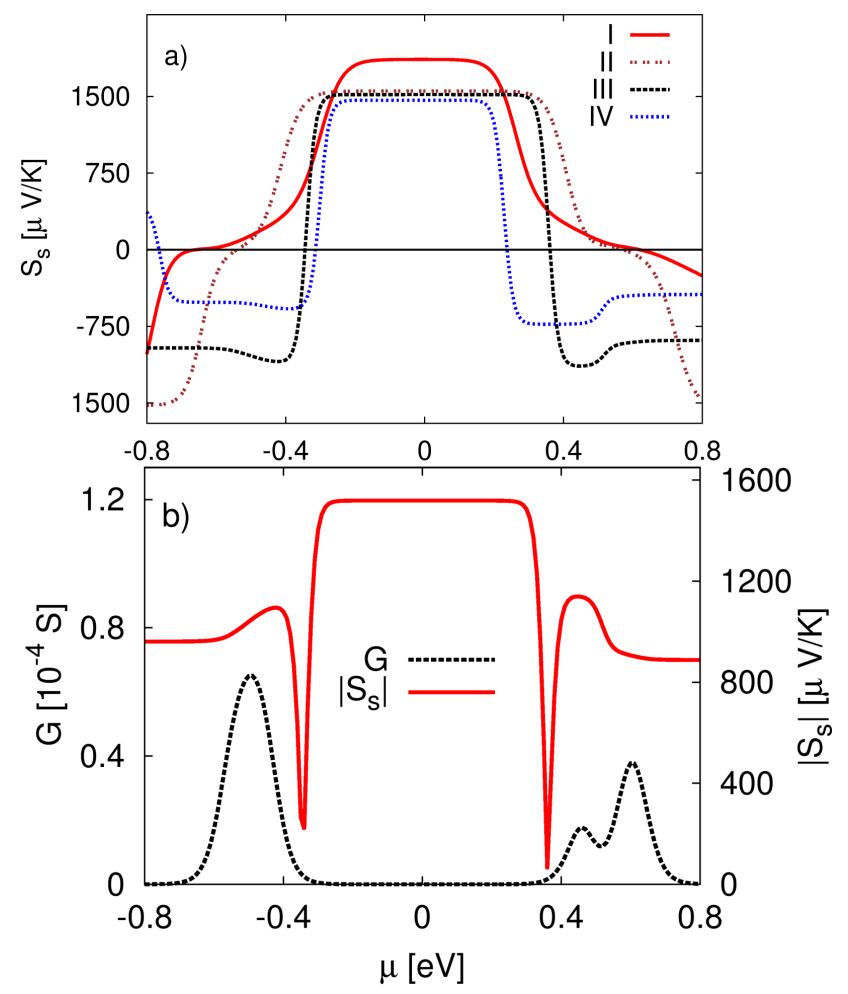

Fig. 4. Spin Seebeck coefficient $S_{s}$ for $T=300 \mathrm{~K}$ of radical chains (a), G and $\left|S_{s}\right|$ vs $\mu$ for $T=300 \mathrm{~K}$ of structure III (b).

the Fermi-Dirac function and lead to a wide central maximum in $S_{s}$ (see also Ref. [15]). In this region of chemical potential the spin Seebeck coefficient rather weakly depends on the type of structure. However, for higher values of $|\mu|$ this dependence is strong. Namely, in the case of structures I and II Seebeck coefficient changes rather slowly with $\mu$ and it remains low in a wide region of $|\mu|$ values greater than $0.4 \mathrm{eV}$. Quite different behavior can be observed for chains of III and IV types. The very rapid changes appear in $S_{s}$ for $|\mu|$ smaller, but close to $0.4 \mathrm{eV}$. The Seebeck coefficient rapidly decreases, changes the sign and at first $\left|S_{s}\right|$ rapidly increases and then, it remains practically constant in the wide region of chemical potential. It is a consequence of the band structure with a number of very flat spin polarized bands leading to narrow peaks in transmission separated by narrow energy gaps (Fig. 2d), which can not be well resolved at elevated temperatures because of broadening of FermiDirac distribution function. Accordingly, $\left|S_{s}\right|$ is strongly enhanced for different values of chemical potential below and above the Fermi level. It is suppressed only in a very narrow region of $|\mu|$. Moreover, according to Fig. $4 \mathrm{~b}$ one can see that in the structure of type III there are regions of chemical potential where both spin polarized conductance and spin Seebeck coefficient simultaneously assume high values leading to considerable enhancement of spin power factor, which strongly enhances thermoelectric spin efficiency.

\section{Conclusions}

One can see that due to a special type of functionalization of one-dimensional carbon structure with NO groups an engineering of the band structure can be achieved which results in a considerable enhancement of spin Seebeck coefficient in a very wide region of chemical potential, and what is more important the electrical conductance exhibits high peaks in these regions of $\mu$. Such an behavior is crucial in view of potential applications in spintonic devices because the systems could act an efficient generators of spin voltage.

\section{Acknowledgments}

Numerical calculations were supported in part by PLGrid Infrastructure.

\section{References}

[1] M. Walter, J. Walowski, V. Zbarsky, M. Munzenberg, M. Schafers, D. Ebke, G. Reiss, A. Thomas, P. Peretzki, M. Seibt,J. S. Moodera, M. Czerner, M. Bachmann, Ch. Heiliger, Nature Mater. 10, 742 (2011).

[2] K. Uchida, S. Takahashi, K. Harii, J. Ieda, W. Koshibae, K. Ando, S. Maekawa, E. Saitoh, $\mathrm{Na}$ ture 455, 778 (2008).

[3] Y. Dubi, M. Di Ventra, Phys. Rev. B 79, 081302(R) (2009).

[4] R. Swirkowicz, M. Wierzbicki, J. Barnaś, Phys. Rev. B 80, 195409 (2009).

[5] K. Zberecki, M. Wierzbicki, R. Swirkowicz, J. Barnaś, J. Phys. Cond. Matt. 29, 045303 (2017).

[6] M. Wierzbicki, R. Swirkowicz, Phys. Rev. B 82, 165334 (2010).

[7] T. Ishida, K. Iwamura, J. Am. Chem. Soc. 113, 4238 (1991).

[8] T. Suga, Y.-J. Pu, K. Oyaizu, H. Nishide, Bull. Chem. Soc. Jpn. 77, 2203 (2004).

[9] M. Kinoshita, Jpn. J. Appl. Phys. 33, 5718 (1994).

[10] N. Tyutyulkov, G. Madjarova, F. Dietz, Mol. Cryst. Liq. Cryst. 305, 249, (1997).

[11] P. Bujak, I. Kulszewicz-Bajer, M. Zagorska, V. Maurel, I. Wielgus, A. Pron, Chem. Soc. Rev. 42, 8895 (2013).

[12] D. Sanchez-Portal, P. Ordejon, E. Artacho, J. M. Soler, Int. J. Quantum Chem. 65, 453 (1997).

[13] M. Brandbyge, J.-L. Mozos, P. Ordejon, J. Taylor, K. Stokbro, Phys. Rev. B 65, 165401 (2002).

[14] J. P. Perdew, K. Burke, M. Ernzerhof, Phys. Rev. Lett. 77, 3865 (1996).

[15] K. Zberecki, R. Swirkowicz, Phys. Status Solidi B 254, 1600783 (2017. 